\title{
Enhanced performance of direct contact membrane distillation via selected electrothermal heating of membrane surface
}

\author{
Farah Ejaz Ahmed ${ }^{a}$, Boor Singh Lalia ${ }^{a}$, Raed Hashaikeh*a, Nidal Hilal*a,b \\ a NYUAD Water Research Center, New York University Abu Dhabi, P.O. Box 129188, Abu Dhabi, \\ United Arab Emirates \\ ${ }^{b}$ Centre for Water Advanced Technologies and Environmental Research (CWATER), College of \\ Engineering, Swansea University, Fabian Way, Swansea SA1 8EN, UK \\ * n.hilal@swansea.ac.uk \\ * raed.hashaikeh@nyu.edu
}




\begin{abstract}
Membrane distillation (MD) is a thermally driven separation process with great potential, but is currently limited by low energy efficiency. Heating of the entire circulating feed represents a major source of energy consumption in MD. Here, we present electrically conductive carbon nanostructure (CNS-) coated polypropylene (PP) membranes as a possible candidate to mitigate energy consumption through selected electrothermal heating of the membrane surface. A membrane for MD was coated with CNS using a tape casting technique. The resulting CNS-PP membrane is hydrophobic, and its smaller pore size and narrow pore size distribution resulted in a higher liquid entry pressure compared to the uncoated PP membrane. An increase in surface temperature was observed when a current was passed through the conductive CNS layer. The CNS layer on the PP membrane acts as an electrothermal heater when an AC potential is applied, and the rate of heating is proportional to the amplitude of applied AC potential. We applied electrothermal heating of these membranes to desalination by direct contact membrane distillation, in conjunction with heating of the circulating feed, and compared the performance with and without application of $A C$ bias at three feed temperatures viz. 40,50 and $60^{\circ} \mathrm{C}$. Applying a potential across the CNS layer increased permeate flux by 75,76 and $61 \%$ at feed temperatures of 40,50 and $60{ }^{\circ} \mathrm{C}$ respectively, while maintaining a salt rejection of $>99 \%$. This increase in flux is accompanied by a reduction in specific energy consumption of greater than $50 \%$ for all three feed temperatures. By combining electrothermal surface heating with $M D$, this study paves the way for smart, low-energy MD systems.
\end{abstract}

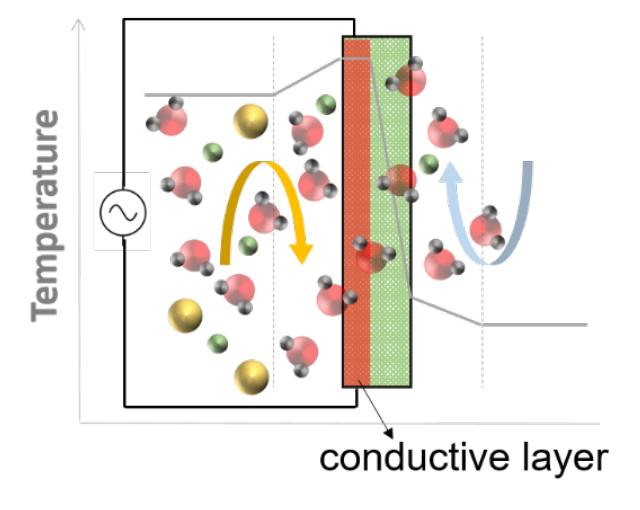

4 Cold circulating permeate

Dot circulating feed

Membrane

v $\mathrm{H}_{2} \mathrm{O} \mathrm{Cl}^{-}$

- $\mathrm{Na}^{+}$ 
Keywords: membrane distillation, desalination, conductive membrane, electrothermal heating

\section{Introduction}

Membrane distillation (MD) is a thermally driven desalination process that can be applied to a range of feed types, from brackish water to highly saline brines. Its compactness, modularity and ability to separate at lower temperatures offer an advantage over other thermal desalination processes [1]. Compared to more widely used reverse osmosis (RO) desalination, MD has a lower propensity of membrane fouling and is more suitable for highly saline feeds [2, 3]. However, the low energy efficiency of MD as compared to RO still deters its application as a large-scale desalination process [4].

In the simplest configuration of MD, known as direct contact membrane distillation (DCMD), a porous hydrophobic membrane separates a high-temperature feed and low-temperature permeate. This temperature difference across the membrane causes a partial vapor pressure gradient which in turn allows vapor to form on the hot surface of the membrane [5], pass through the membrane from the feed to the permeate side and condense on the cooler surface of the membrane. Efforts in MD have largely involved development of new and modified MD configurations as well as novel membrane materials to lower energy cost. However, heating of the circulating feed still represents the largest portion of energy consumption in MD at present [6].

To tackle this challenge, researchers have recently directed their attention to localized heating, i.e. heating near/at the membrane surface. If the membrane-feed interface could be heated directly, energy costs can potentially be lowered. Some researchers have embedded photothermal particles in membranes and coatings [7-13], to induce heating via the thermoplasmonic effect $[14,15]$. Alsaati and Marconnet used an electric heater to heat the region of the fluid near the surface of a silver membrane for air gap MD [16]. Although the high thermal conductivity of the membrane is suitable for uniform heating, it also poses the risk of large conductive losses through the membrane.

Electrothermal heaters provide a simple form of direct heating in which a current passing through the film causes a rise in temperature. Graphene [17] and carbon nanotube (CNT) [18] film heaters have shown promising electrothermal behavior in air. Very few researchers have assessed the use of CNT membranes for electrothermal heating in MD, owing to the instability of the film in ionizable media such as water, under the effect of an electric field. Dudchenko et al. point out that a DC voltage is not adequate to heat these films in water [19]. Direct surface heating reduces the unwanted effects of temperature polarization [4]. Li et al. [20] devised a reverse Joule- 
heating air gap $\mathrm{MD}$ in which reduced graphene oxide was coated on a commercial polytetrafluoroethylene (PTFE) membrane, but the conductive layer was placed at the air gap, not on the feed side of the membrane. Although they argue that this opposite temperature gradient prevents wetting, the flux through the membrane is very low. Both these studies were carried out on cells with dimensions in the millimeter range and rely solely on electrothermal membrane heating. However, given the high energy consumption in both cases, it may be beneficial to combine electrothermal surface heating with external heating of the circulating feed. This would also reduce the amount of high quality electrical energy required for surface heating, as low-grade heat can still be applied to heat the circulating feed. In this work, carbon nanostructures (CNS) were coated on the surface of a commercial polypropylene (PP) membrane and subjected to electrothermal heating to assist in DCMD. We demonstrate control over the morphology, pore size distribution, porosity and electrical properties of the coated membrane. We demonstrate the effect of applied voltage on the electrothermal heating behavior of the membranes. Finally, we apply these membranes to DCMD with heating of the circulating feed, comparing performance in terms of flux and energy consumption, with and without the application of AC bias.

\section{Materials and methods}

Carbon Nanostructures (average length $\sim 300 \mathrm{~nm}$ and diameter 5- $10 \mathrm{~nm}$ ) were obtained from Applied NanoStructured Solutions LLC (ANS). CNS consist of entangled carbon nanotubes with high electrical conductivity and large surface area [21]. Ethanol absolute was obtained from VWR International. Commercial polypropylene (PP) membrane with mean pore size of $0.2 \mu \mathrm{m}$ was purchased from Sterlitech.

\subsection{Fabrication of CNS-PP membranes}

To coat the PP membrane with CNS, CNS flakes were dispersed in a 1:1 (v/v) ethanol/DI water according to a procedure developed by Susantyoko et al. [22]. In this procedure, $400 \mathrm{mg}$ of CNS were first mixed into $10 \mathrm{ml}$ each of DI water and ethanol for 2 minutes using a mortar and pestle. $90 \mathrm{ml}$ of DI water and $90 \mathrm{ml}$ of ethanol were then added to the mixture, which was simultaneously stirred at 1000 RPM and sonicated using a probe sonicator (QSonica Q700) at 40\% amplitude for 10 minutes. The resulting ink-like suspension was degassed in a desiccator under vacuum for 4 hours. Two graphite electrodes (MinGraph Flexible graphite) were pasted onto the membrane using Araldite $A B$ epoxy adhesive. To coat the membrane, a mask was prepared such that CNS was only coated on the active membrane area. This was done to prevent uneven heating of the membrane as the region outside the active area is exposed to air. Heat dissipation through water causes different heating behavior in air and in water. The suspension was coated using a doctor blade technique. The coating height and speed were kept at $2.5 \mathrm{~mm}$ and $40 \mathrm{~mm} / \mathrm{min}$, 
respectively. The membrane was then dried at $120^{\circ} \mathrm{C}$ for 1 hour before characterization. Figure 1 shows a schematic of the membrane fabrication technique.

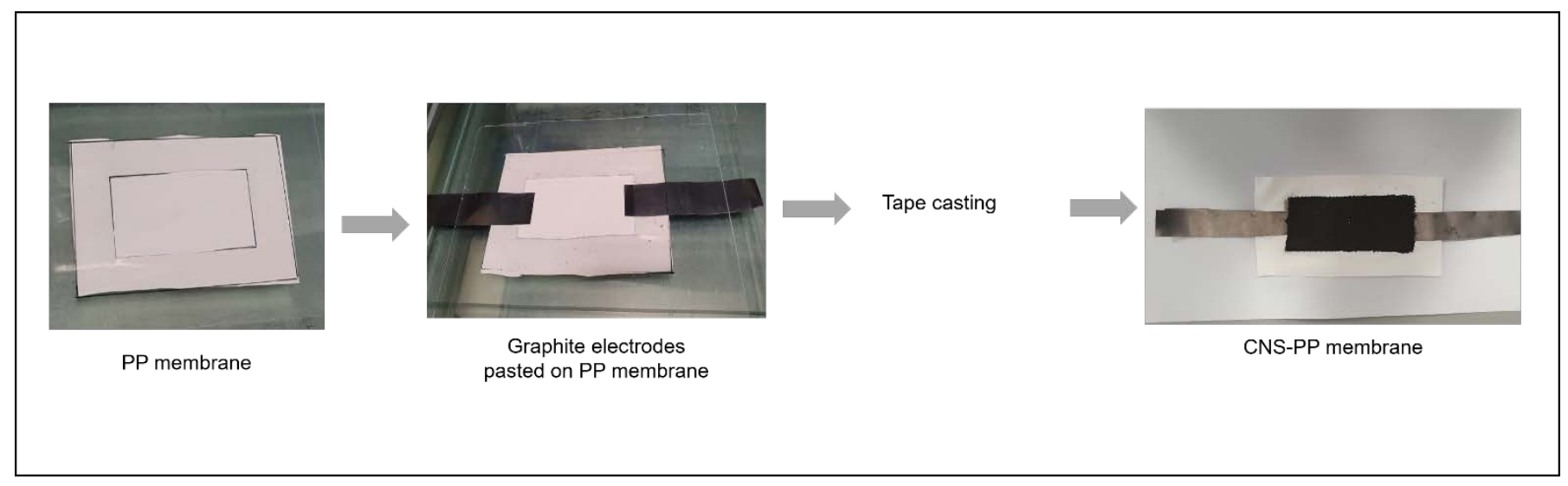

Figure 1: Fabrication of CNS-coated PP membrane

\subsection{Membrane characterization}

\subsubsection{Morphology}

The morphology of the uncoated and coated membranes was examined using high-resolution scanning electron microscopy (FEI Quanta 450 FEG, Netherlands) under high vacuum. For crosssectional imaging, samples were dipped in liquid nitrogen to freeze their structure and broken. The non-conductive uncoated PP membrane was coated with a thin layer of Au using the 108 Auto Sputter Coater (Ted Pella, USA) prior to imaging.

\subsubsection{Contact angle}

Contact angle measurements were taken on the membrane surface before and after coating with CNS, in a controlled temperature environment using an EasyDrop Standard drop shape analysis (DSA100, Krüss GmbH, Germany). A digital image was taken as a $4 \mu \mathrm{L}$ droplet of DI water was generated on the membrane surface using a syringe with needle diameter $0.51 \mathrm{~mm}$. Measurements were carried out at temperatures varying between RT and $70{ }^{\circ} \mathrm{C}$. The software was then used to determine the contact angle using the sessile drop method. The contact angle was measured three times for each sample and the average was recorded.

\subsubsection{Pore size, porosity and liquid entry pressure}

The pore size distribution of the uncoated and coated membranes was obtained using capillary flow porometry on the PMI iPore 500 (Ithaca, USA). Membrane samples of diameter $2.5 \mathrm{~cm}$ were wetted with a wetting liquid (Galwick, surface tension: 15.9 dynes $\mathrm{cm}^{-1}$ ) and pressurized air was applied in increments to displace the liquid from its pores, to determine the pore size distribution. 
Porosity measurements were carried out by wetting samples of known dimensions with Siwick묘 (surface tension: 20.1 dynes $\mathrm{cm}^{-1}$ ). Excess liquid was removed from the surface of the membrane. The dry and wet mass of the sample were measured and the pore volume was determined. Porosity was calculated as the ratio of pore volume to sample volume.

Liquid entry pressure measurements (LEP) carried out on the iPore 500 (PMI, Ithaca, USA). This time, the membrane sample was covered with DI water and pressurized air was applied in increments. The bubble point pressure, in this case referring to the pressure at which water first permeated through the membrane, was recorded as the LEP of water. At least two samples of each membrane were used to determine porosity and LEP.

\subsubsection{Electrical conductivity}

Electrical resistivity was measured using a four point probe technique, according to the van der Pauw method[23], using the Metrohm Autolab PSTAT302N potentiostat and the Autolab Microcell HC. A square sample of $1 \mathrm{~cm} \times 1 \mathrm{~cm}$ was placed inside the measuring cell. The sample was in contact with four gold probes around its perimeter. The probes are labeled 1-4 from the top left in the anticlockwise direction. A current is passed between 1 and 2 and then between 2 and 3 , and the voltage is measured across 3 and 4 and then between 4 and 1 respectively. From these measurements, $\mathrm{R}_{12,34}$ and $\mathrm{R}_{23,41}$ are determined, and the electrical conductivity $k_{e l}$ is calculated using the equation below:

$$
\frac{1}{k_{e l}}=\rho=\frac{\pi}{\ln 2} \cdot t \cdot \frac{R_{12,34}+R_{23,41}}{2} \cdot f
$$

where $\rho$ is the electrical resistivity, $\mathrm{t}$ is the thickness of the conductive layer and $f$ is the correction factor corresponding to the ratio $\frac{R_{12,34}}{R_{23,41}}$. The coefficient $\frac{\pi}{\ln 2}$ is known as the van der Pauw coefficient. The thickness of the conductive layer was obtained from cross-section SEM images.

\subsection{Electrothermal heating}

To characterize the electrothermal heating behavior of the membrane, the membrane was immersed in DI water. An AC voltage of either $15 \mathrm{~V}, 24 \mathrm{~V}$ or $30 \mathrm{~V}$ (at $50 \mathrm{~Hz}$ ) was applied using a step-down transformer. The temperature of the membrane surface was recorded using a thermocouple and Labworldsoft 6 software. The time taken for the surface to reach $80{ }^{\circ} \mathrm{C}$ was recorded for all three applied potentials, and temperature vs. time plots were generated to compare their behavior.

\subsection{Membrane distillation}

Performance of the membrane was evaluated using the Convergence Inspector membrane distillation pilot. The active area of the membrane was $7.5 \mathrm{~cm} \times 3 \mathrm{~cm}$. A NaCl solution with TDS 
$10,000 \mathrm{ppm}$ was used as the feed, whereas DI water was used as the permeate. Both streams were recirculated in counter-current directions and flow rates were maintained at $20 \mathrm{~L} / \mathrm{h}$. The mass of the permeate was recorded at 60 s intervals.

For each MD test, the feed was heated to either 40,50 and $60^{\circ} \mathrm{C}$. At each feed temperature, MD tests were carried out with and without applied voltage across the membrane. For electrically assisted heating, a step-down transformer with an AC voltage of $24 \mathrm{~V}$ and a frequency of $50 \mathrm{~Hz}$ was connected to each side of the membrane via graphite electrodes. Each MD test was carried out for 10 hours during which the electrical conductivity of both streams, temperatures, flow rates and permeate mass were recorded. The $A C$ current through the membrane was monitored on a digital multimeter. The permeate was kept at $15^{\circ} \mathrm{C}$.

Permeate flux was calculated from the permeate mass according to the following equation:

$J\left(\mathrm{~kg} \mathrm{~m}^{-2} \mathrm{~h}^{-1}\right)=\frac{\text { (change in permeate mass) }}{\mathrm{t} * A_{m}}$ (1)

where $\mathrm{t}$ is the time and $A_{m}$ is the active membrane area $\left(22.5 \mathrm{~cm}^{2}\right)$, respectively.

Salt rejection (SR) was calculated from feed and permeate conductivities according to the following equation:

$\mathrm{SR}(\%)=\frac{k_{\text {feed }}-k_{\text {permeate }}}{k_{\text {feed }}} \cdot 100(2)$

where $k_{\text {feed }}$ and $k_{\text {permeate }}$ are the conductivities of the feed and permeate respectively.

\section{Thermal efficiency and energy consumption}

Thermal efficiency (TE) and specific energy consumption (SEC) were calculated as performance indicators for MD.

The thermal efficiency was calculated as follows [24, 25]:

$T E(\%)=\frac{Q_{v a p}}{Q_{v a p}+Q_{c o n d}}=\frac{J A_{m} \Delta H_{v}}{Q_{m}} \times 100$

where $J$ is the permeate flux through the membrane in $\mathrm{kg} \mathrm{m}^{-2} \mathrm{~h}^{-1}, A_{m}$ is the active area of the membrane in $\mathrm{m}^{2}, \Delta H_{v}$ is the enthalpy of vaporization of water in $\mathrm{kJ} / \mathrm{kg}$ and $Q_{m}$ is the heat flux through the membrane in $\mathrm{kW}$. $Q_{\text {cond }}$ is the heat lost due to conduction through the membrane $(\mathrm{kW})$ and $Q_{v a p}$ is the latent heat associated with vaporized molecules $(\mathrm{kW})$.

By energy conservation, the total heat flux through the membrane equals the heat lost by the feed as it is circulated through the membrane. $Q_{m}$ can be calculated as:

$Q_{m}=\dot{m}_{f} C_{p}\left(T_{f, \text { in }}-T_{f, \text { out }}\right)$ 
where $\dot{m}_{f}$ is the mass flow rate in $\mathrm{kg} \mathrm{s}^{-1}, C_{p}$ is the feed water specific heat $\left(\mathrm{kJ} \mathrm{kg}^{-1}{ }^{\circ} \mathrm{C}^{-1}\right), T_{f, \text { in }}$ and $T_{f, \text { out }}$ are the inlet and outlet feed temperatures respectively.

Specific energy consumption (SEC) was calculated in $\mathrm{kWh} \mathrm{m}^{-3}$ from the following equation [24, 26]:

$S E C=\frac{Q_{m} \rho}{J A_{m}}(5)$

where $\rho$ is the density of water in $\mathrm{kg} \mathrm{m}^{-3}$.

\section{Results and Discussion}

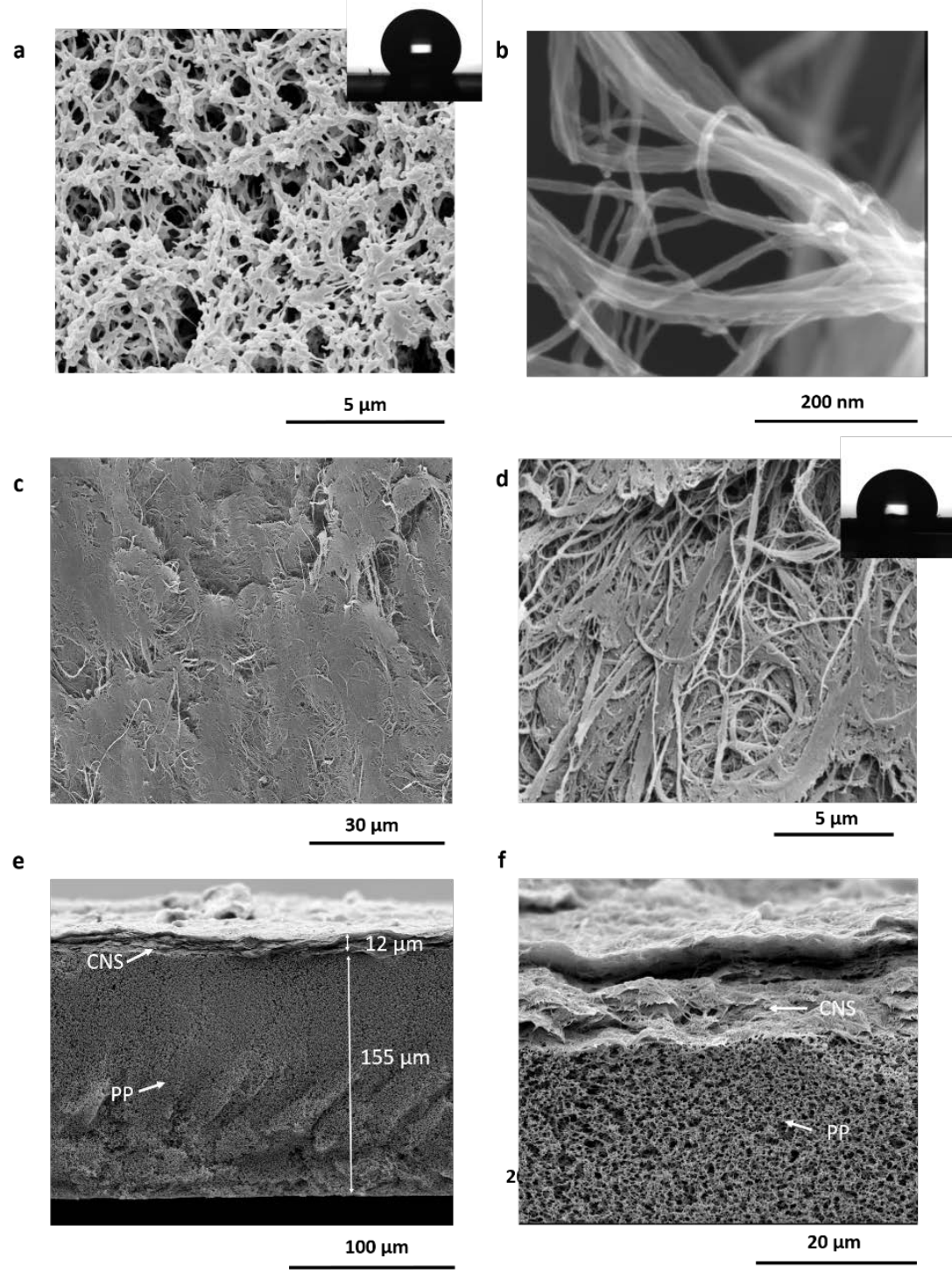

Figure 2: Scanning electron microscope (SEM) images of (a) pristine polypropylene (PP) membrane, (b) CNS, and (c, d) CNS-coated PP (CNS-PP) membrane; SEM images of the cross- 
section of CNS-PP at different magnifications (e, f). The insets of Fig. 2a and d show a water droplet on the surface of PP and CNS-PP membrane, respectively.

SEM images of the pristine and coated PP membrane (Figure 2) show that the membranes have dissimilar surface morphologies. The former exhibits large sponge-like pores, whereas the coated membrane is covered with randomly oriented, highly entangled fibrous CNS. The pores on the surface of the CNS-coated membrane also appear smaller. A CNS layer is also visible in the crosssectional SEM image (Fig. 2f). CNS used in this study has a 3D interconnected structure with tube diameter of 6-9 nm and length of 150-300 microns. Our previous study [27] shows that free standing CNS films have a tensile strength of $\sim 4 \mathrm{MPa}$. The entangled microstructure of CNS (Fig $2 b$ ) enables it to be anchored inside the membrane pore structure and form a stable layer after being solution cast on the PP membrane surface. The thickness of the CNS layer is $12 \pm 2 \mu \mathrm{m}$.

Modification of the commercial membrane results in a lower apparent contact angle for water droplets, as shown in Figure 3. Although the contact angle of the membrane drops from $133^{\circ}$ to $104^{\circ}$ upon modification with CNS, the CNS-coated membrane is still hydrophobic. Additionally, the more hydrophobic PP layer below CNS will provide resistance to wetting. Liquid entry pressure also increases with the CNS, as discussed in the following section. Membrane distillation relies on the use of hydrophobic membranes to prevent transfer of feed in the liquid phase. As the temperature is varied between 20 and $70{ }^{\circ} \mathrm{C}$, there is little change in the apparent water contact angle on both membranes, with contact angles ranging between $119^{\circ}$ and $137^{\circ}$ for $\mathrm{PP}$, and $100^{\circ}$ and $112^{\circ}$ for CNS-PP. This is supported by a recent study by Villa et al. [28], who found that there is no significant change in the water contact angle with temperature for hydrophobic surfaces, according to the decreasing trend model (DTm). A survey of literature shows that the temperature dependence of surface wettability however has been a subject of scrutiny and disagreement for decades now. Phillips and Riddiford first suggested that the temperature coefficient of contact angles is very small, indicating that the effect of temperature on the surface tension and adhesion to solid may balance each other out [29,30]. Study of the temperature effect of water contact angle specifically on carbon nanotubes has thus far been limited to molecular dynamics simulations and single walled carbon nanotubes (SWCNTs) [31, 32]. 


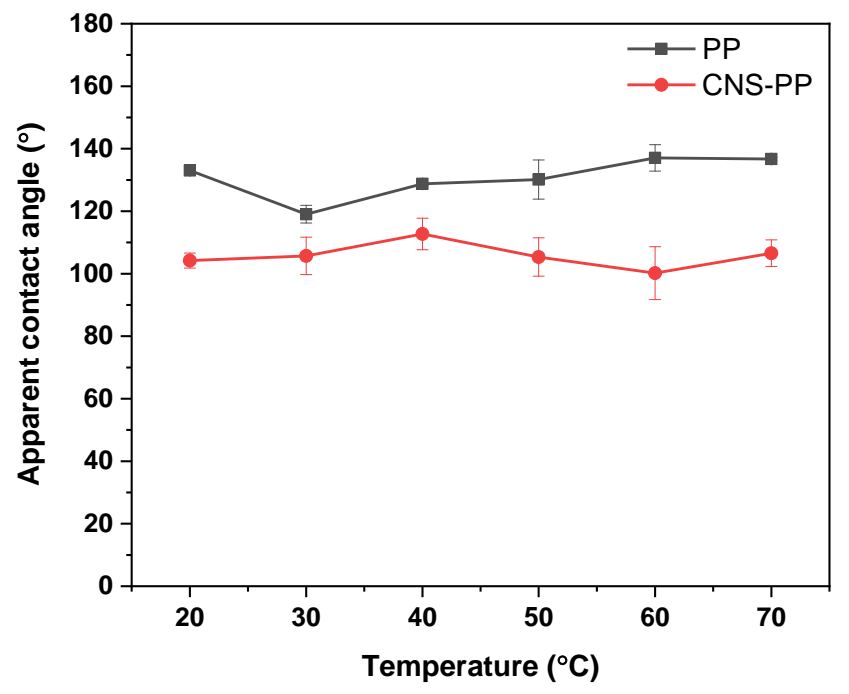

Figure 3: Apparent contact angle of water on the pristine PP and CNS-coated PP membranes at different temperatures. Error bars represent standard deviation from three independent measurements.

The pore size distributions of the pristine PP membrane and CNS-coated membrane were measured with a capillary flow porometer, and are shown in Figure 4. Key parameters such as mean pore size, bubble point, porosity and LEP are shown in Table 1. Upon coating with CNS, the mean pore size decreased from $0.19 \mu \mathrm{m}$ to $0.034 \mu \mathrm{m}$. The bubble point, i.e. the largest pore size, decreased from $0.78 \mu \mathrm{m}$ to $0.36 \mu \mathrm{m}$. The mean pore size of the CNS-coated PP membrane is an order of magnitude lower than that of commercial PP (Table 1). Although the observed mean pore size is slightly lower than the appropriate pore size range of 0.1 to $1 \mu \mathrm{m}$ recommended for MD membranes [33,34], the CNS layer here is added to demonstrate a proof-of-concept on electrothermally assisted heating for MD, and the pore size can be tuned in future studies. Additionally, the narrower pore size distribution of the CNS-coated membrane compared to the commercial membrane is desirable for MD [34]. 


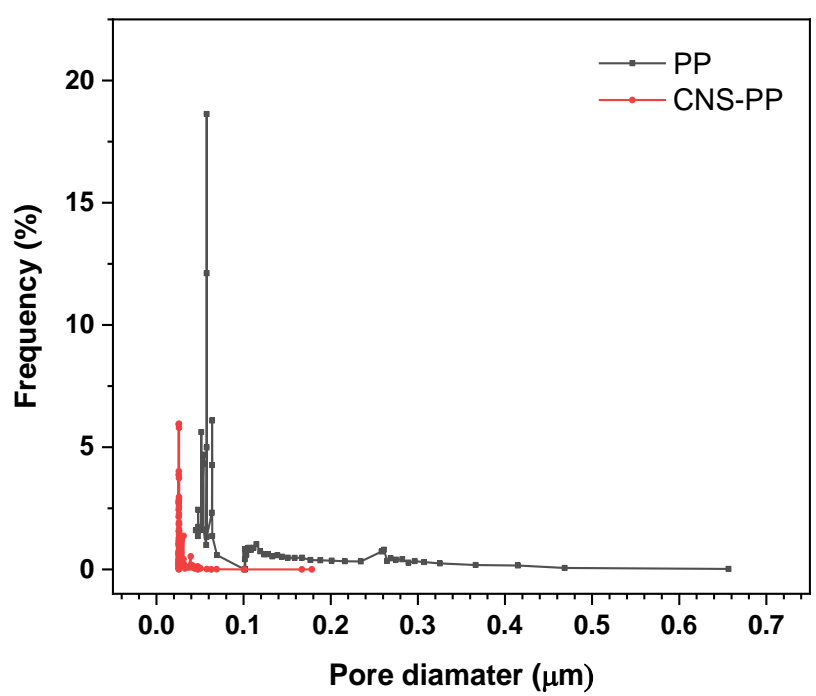

Figure 4: Pore size distribution of PP and CNS-PP membranes

Table 1: Parameters of PP and CNS-PP membrane

\begin{tabular}{|l|c|c|}
\hline & PP & CNS-PP \\
\hline Bubble point $(\mu \mathrm{m})$ & 0.776 & 0.3576 \\
\hline Mean pore size $(\mu \mathrm{m})$ & 0.1931 & 0.0341 \\
\hline Porosity $(\%)$ & $85 \pm 0.4$ & $81 \pm 1.7$ \\
\hline LEP (bar) & $2.25 \pm 0.004$ & $2.75 \pm 0.02$ \\
\hline
\end{tabular}

Porosity and LEP results are shown in Figure 6a. LEP is a key membrane characteristic for MD. It determines the minimum transmembrane pressure required for a liquid, in this case water, to penetrate into a membrane pore [35]. As such, a high LEP is required for MD to resist wetting and feed penetration through the membrane. The LEP of hydrophobic membranes follows the Laplace equation[36, 37], described as: $L E P=\frac{4 \gamma}{d_{P}}|\cos \theta|$, where $\gamma$ is the surface tension of water $\left(72\right.$ dynes $\left.\mathrm{cm}^{-1}\right), d_{P}$ is the mean pore size and $\theta$ is the water contact angle. The LEP of the CNS-coated membrane is $2.75 \pm 0.02$ bars, while that of the commercial PP membrane is $2.25 \pm$ 0.004 bars the commercial PP membrane. Despite lower contact angle, the increase in LEP of the CNS-coated membrane is driven by the reduced pore size and tighter pore size distribution. The porosity of both commercial and CNS-coated PP membranes was maintained at $>80 \%$.

Electrical conductivity of the CNS-coated membrane was measured using the van der Pauw method. Measurements were performed as a function of temperature from 20 to $70{ }^{\circ} \mathrm{C}$ and the results are shown in Figure 6b. At room temperature, the CNS-PP membrane exhibits an electrical conductivity of $18.4 \pm 2.2 \mathrm{~S} \mathrm{~cm}^{-1}$. This is on the same order of magnitude as values found in literature for buckypaper membranes, prepared from a dispersion of randomly oriented CNTs [38-41], MWCNT composites [42, 43] and MWCNT yarns [44]. However, this is significantly lower 
than reported $k_{e l}$ values of $10^{2}-10^{5} \mathrm{~S} \mathrm{~cm}^{-1}$ for individual CNTs and CNT bundles [45, 46], due to the presence of a large number of defects and entanglements in CNS [21], as well as the high porosity of the CNS-PP membrane.

a

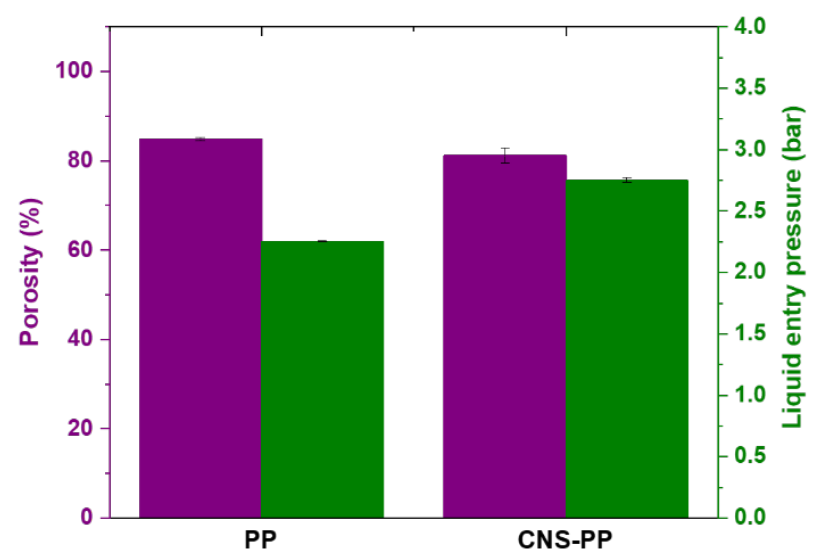

b

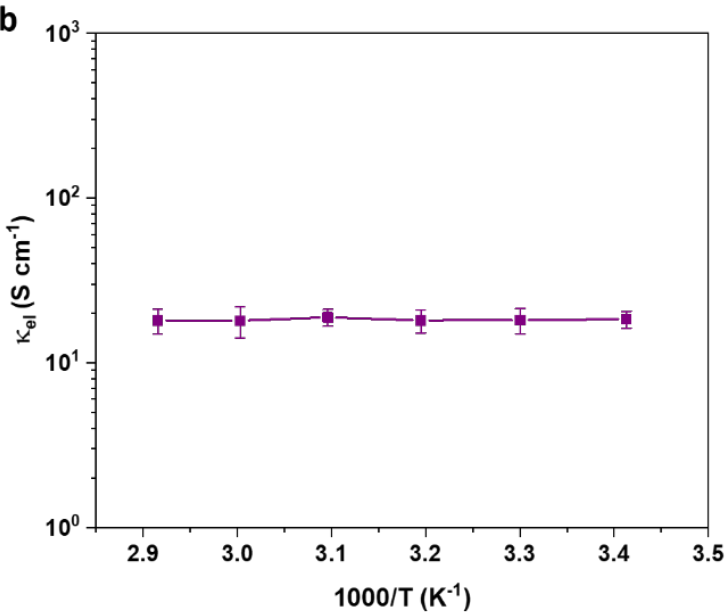

Figure 5: a) Porosity and liquid entry pressure of PP and CNS-coated PP membrane; b) Effect of temperature on electrical conductivity of CNS-coated PP membrane.

Electrical conductivity was measured in the region of interest, between 20 and $70{ }^{\circ} \mathrm{C}$, at $10{ }^{\circ} \mathrm{C}$ intervals. Within this range, Arrhenius behavior is observed i.e. $\log k_{e l}$ vs. 1000/T yields a linear relationship. The horizontal line indicates a very weak temperature dependence, and metalliclike behavior, with zero activation energy. Similar behavior of electrical conductivity has previously been observed for buckypapers in this temperature region $[41,47]$.

The electrothermal heating behavior was observed by immersing the membrane in water and applying an AC potential of either 15, 24 and 30V. The temperature was recorded at 10 second intervals until the surface temperature reached $80^{\circ} \mathrm{C}$. Heating was observed for all three applied potentials, with an increase in the rate of heating with applied potential, as shown in Figure 7. It took the membrane surface 16,9 and 2 minutes to reach a surface temperature of $80^{\circ} \mathrm{C}$ at 15,24 and $30 \mathrm{~V}$ respectively. As current is passed through the conductive layer of the membrane, the resistance to electron flow causes it to heat [4]. Although rapid heating was observed at a potential of $30 \mathrm{~V}$, it caused localized melting of the PP membrane. For this reason, $24 \mathrm{~V}$ was chosen as appropriate voltage to assist in MD. 


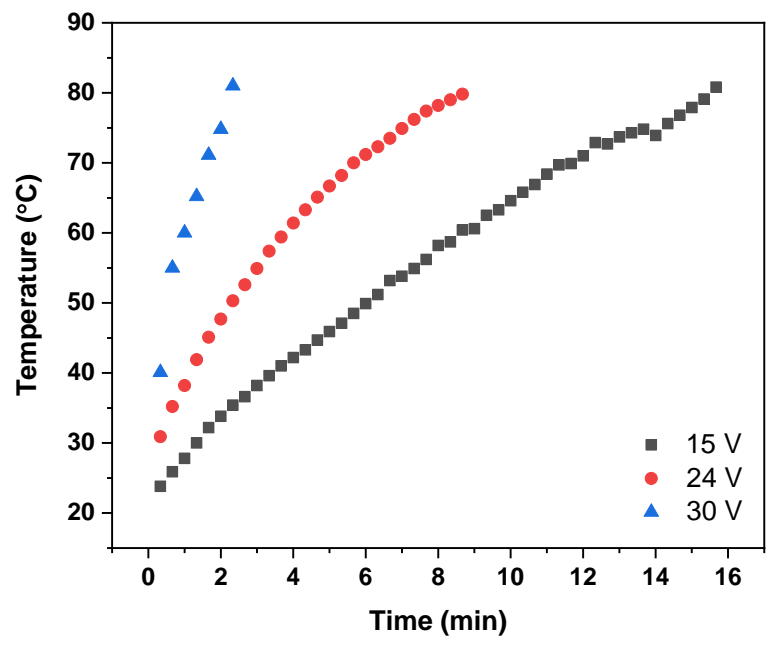

Figure 6: Membrane surface temperature with time for different AC potentials in water.

For MD tests, the feed was circulated at 40,50 and $60^{\circ} \mathrm{C}$. At each of these temperatures, MD was operated with and without $24 \mathrm{~V}$ AC potential to evaluate the effect of applied potential on MD performance. Figure 8 shows the permeate mass flux through the membrane with and without applied potential, at the three feed temperatures. In all cases, flux through the membrane increased significantly when a potential was applied. For feed temperatures of 40 and $50{ }^{\circ} \mathrm{C}$, the applied potential enhanced the average flux by $>75 \%$, whereas at a feed temperature of $60{ }^{\circ} \mathrm{C}$, a $61 \%$ improvement in flux was observed between no potential and $24 \mathrm{~V}$. Electrothermal heating of the membrane surface with applied potential reduces the effects of temperature polarization on the feed side. This keeps up the vapor pressure difference across the membrane, which in turn causes more vapor to form and pass through the membrane. 


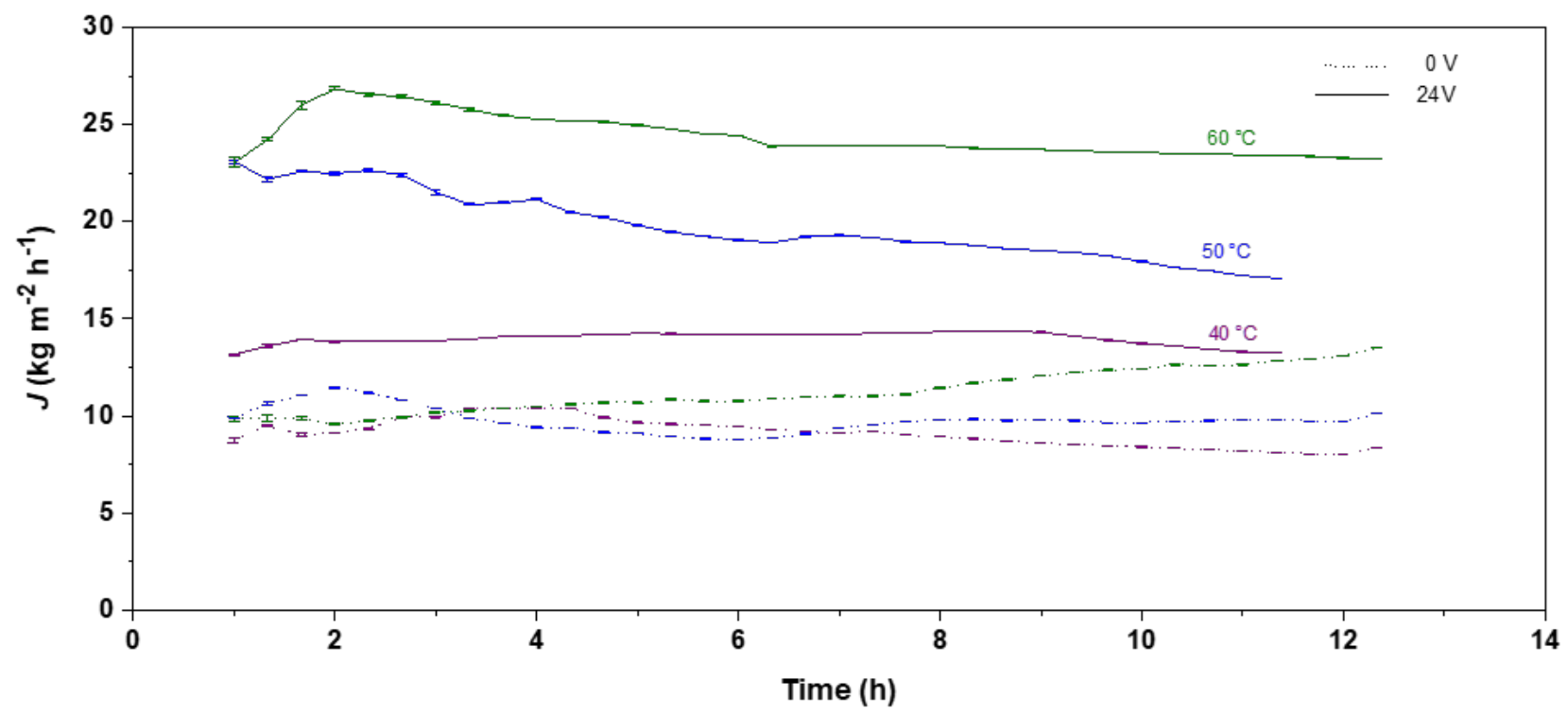

Figure 7: Flux through CNS-PP membrane at feed temperatures of $40^{\circ} \mathrm{C}$ (purple), $50^{\circ} \mathrm{C}$ (blue) and $60^{\circ} \mathrm{C}$ (green), with and without an applied $\mathrm{AC}$ potential

However, in some cases, it was noted that the flux declined after several hours. As the process is run for 12 hours, the concentration of $\mathrm{NaCl}$ near the surface of the membrane rises as pure water vapor passes through the membrane. This concentration polarization reduces the driving force and therefore the flux through the membrane [48]. Table 2 shows the average flux and salt rejection rate for each feed temperature, as well as the current passing through the membrane under the effect of potential. The membrane retains a high separation efficiency of greater than $99 \%$ in all tests.

Table 2: Average flux and salt rejection for CNS-PP membrane

\begin{tabular}{|c|c|c|c|c|}
\hline $\mathbf{T}\left({ }^{\circ} \mathbf{C}\right)$ & Potential (V) & Current (A) & $\mathbf{J}\left(\mathbf{k g ~ m}^{-\mathbf{2}} \mathbf{h}^{-\mathbf{1}}\right)$ & $\mathbf{S R}(\%)$ \\
\hline \multirow{2}{*}{40} & 0 & - & 7.9 & 99.8 \\
\cline { 2 - 5 } & 24 & 2.4 & 13.8 & 99.8 \\
\hline \multirow{2}{*}{50} & 0 & - & 9.7 & 99.5 \\
\cline { 2 - 5 } & 24 & 2.4 & 17.0 & 99.5 \\
\hline \multirow{2}{*}{60} & 0 & - & 14.2 & 99.5 \\
\cline { 2 - 5 } & 24 & 2.1 & 22.9 & 99.2 \\
\hline
\end{tabular}

Figure 9 shows the effect of applied potential and feed temperature on average flux, thermal efficiency and specific energy consumption. Although increasing the feed temperature increases productivity, this comes at the cost of lower thermal efficiency and greater energy consumption. As the temperature is increased, there is a greater difference between the feed and ambient 
temperature causing more heat to be lost to the environment. Elmarghany et al. recently reported similar findings in their study of the thermal analysis of DCMD systems[24]. Our results show that an SEC reduction of greater than $50 \%$ is observed between $\mathrm{OV}$ and $24 \mathrm{~V}$ at all three feed temperatures. Although still relatively high, SEC values obtained are in line with those reported in literature for lab-scale DCMD systems [49]. SEC can be further reduced by using higher flow rates, increasing the size of the system and/or using heat recovery devices [19, 50].
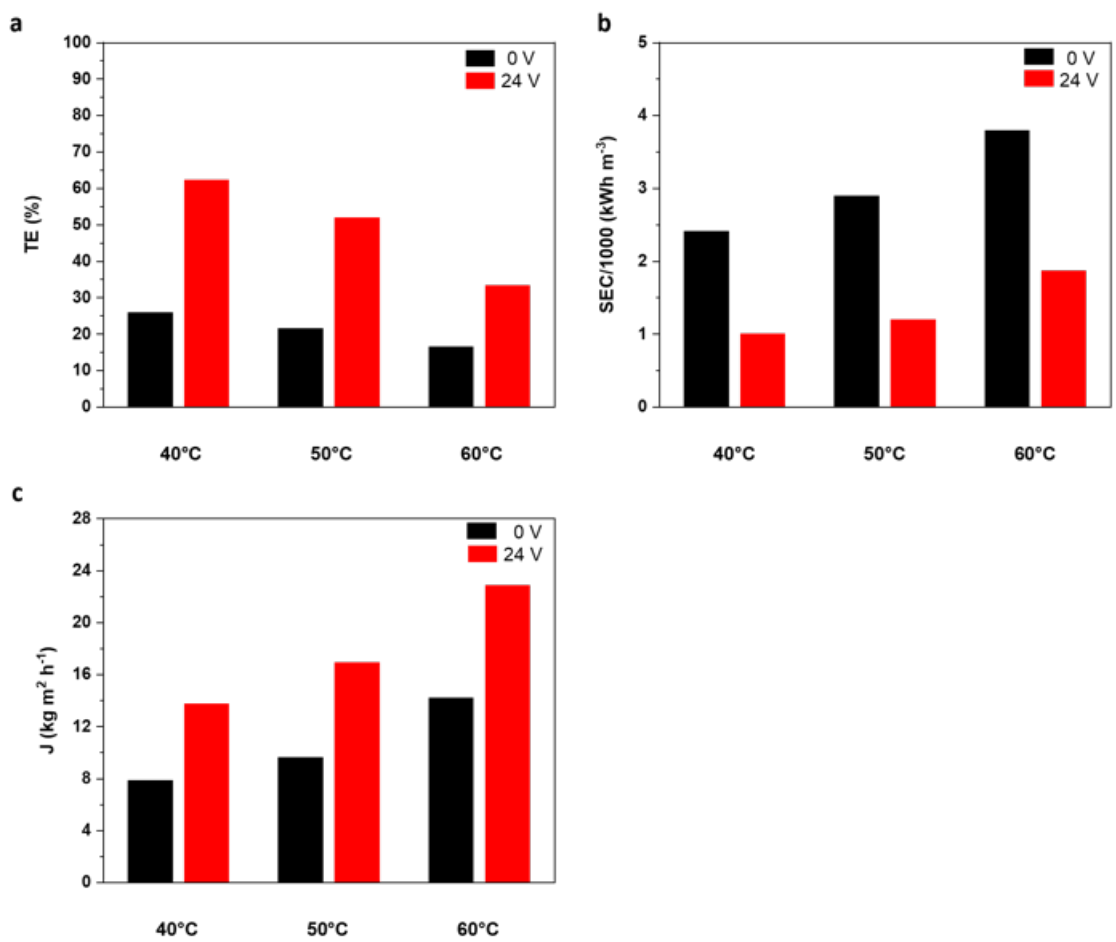

Figure 8: Comparison of (a) thermal efficiency, (b) specific energy consumption and (c) permeate flux for CNS-PP membrane with and without applied potential at different feed temperatures

It is known that increasing the feed temperature and therefore the temperature gradient across the membrane leads to an increase in permeate flux [51]. Increasing the feed temperature from $40^{\circ} \mathrm{C}$ to $60^{\circ} \mathrm{C}$ with no potential leads to a flux increase of $81 \%$, while applying a $24 \mathrm{~V}$ potential to the membrane when the feed temperature is kept at $40^{\circ} \mathrm{C}$ causes $75 \%$ increase in flux. For similar flux improvement, the former translates into a $58 \%$ increase in SEC, while the use of electrothermal membrane heating results in 58\% energy savings. Electrothermal heating of the membrane surface not only increases productivity, but also enhances thermal efficiency by more than two fold, for all feed temperatures.

This demonstrates the potential of electrothermal heating coupled with bulk feed heating to achieve greater productivity at lower costs. The coupling of the two types of heating as carried 
out in this study ensures that the system is still partially driven by waste heat, a point that has long been cited as an advantage of MD over pressure-driven processes [52-55]. Additionally, using the two heat sources together can potentially break the barrier for direct surface heating [4] and allow it to be implemented beyond small-scale systems.

\section{Conclusion}

MD has the potential to compete with pressure-driven desalination processes, due to its ability to treat highly saline feedwater and its lower propensity for fouling. However, the low energy efficiency of MD systems has been a major deterrent. In this work, we modified commercial MD membranes with carbon nanostructures, to introduce electrothermal heating of the membrane surface.

CNS lowers the water contact angle of the PP membrane, however the resulting membrane isstill hydrophobic. Although the mean pore size of the modified membrane is lower than the PP membrane, the modified membrane also has a narrower pore size distribution, while retaining a high porosity of $81 \%$. The modified membrane has a greater liquid entry pressure for water, making it suitable for MD. In the temperature range of 20 to $70^{\circ} \mathrm{C}, \mathrm{CNS}$-PP maintains an electrical conductivity of $18 \mathrm{~S} \mathrm{~cm}^{-1}$.

The CNS-coated membrane acts as an electrothermal heater in water when an AC potential is applied and the rate of heating increases with the amplitude of applied potential. We applied the electrothermal behavior of the CNS-PP membrane to assist in mass transfer during MD process. DCMD tests were carried out at three feed temperatures with and without electrothermal assistance. We found that the application of $24 \mathrm{~V} \mathrm{AC}$ potential resulted in an increase in flux of $75 \%$ at feed temperatures of 40 and $50{ }^{\circ} \mathrm{C}$. This increase is accompanied with a reduction in SEC of $58 \%$. In contrary, although simply increasing the feed temperature also results in enhanced flux, the lower thermal efficiency leads to an increase in SEC. Salt rejection with $10,000 \mathrm{ppm} \mathrm{NaCl}$ feed is maintained at $>99 \%$. Future work includes better control of the current through the conductive layer and further reduction of SEC by optimizing process conditions and applying other MD configurations. We believe that our approach to incorporate electrothermal membrane heating with heating of the bulk feed offers several advantages. First, greater productivity is achieved at significantly lower energy costs. Second, the process does not entirely rely on high quality electricity. Waste heat can still be used, or in fact be used more efficiently, as increasing the feed temperature as a means to enhance flux only leads to lower thermal efficiency. Finally, the coupling of the two heat sources with energy savings can break the barrier that limits direct membrane surface heating to small-scale systems. We envision that this approach will guide the design for smart, energy-efficient MD systems for desalination. 


\section{References}

1. Alkhudhiri, A., N. Darwish, and N. Hilal, Treatment of saline solutions using Air Gap Membrane Distillation: Experimental study. Desalination, 2013. 323: p. 2-7.

2. Wang, W., et al., Trade-off in membrane distillation with monolithic omniphobic membranes. Nature communications, 2019. 10(1): p. 1-9.

3. Minier-Matar, J., et al., Field evaluation of membrane distillation technologies for desalination of highly saline brines. Desalination, 2014. 351: p. 101-108.

4. Deshmukh, A., et al., Membrane distillation at the water-energy nexus: limits, opportunities, and challenges. Energy \& Environmental Science, 2018. 11(5): p. 1177-1196.

5. Alkhudhiri, A., N. Darwish, and N. Hilal, Membrane distillation: A comprehensive review. Desalination, 2012. 287: p. 2-18.

6. Lokare, O.R., et al., Importance of feed recirculation for the overall energy consumption in membrane distillation systems. Desalination, 2018. 428: p. 250-254.

7. Politano, A., et al., Photothermal Membrane Distillation for Seawater Desalination. Advanced Materials, 2017. 29(2): p. 1603504.

8. Dongare, P.D., et al., Nanophotonics-enabled solar membrane distillation for off-grid water purification. Proceedings of the National Academy of Sciences, 2017. 114(27): p. 6936.

9. Tan, Y.Z., et al., Photothermal-enhanced and fouling-resistant membrane for solar-assisted membrane distillation. Journal of Membrane Science, 2018. 565: p. 254-265.

10. $\mathrm{Wu}, \mathrm{X}$., et al., Localized heating with a photothermal polydopamine coating facilitates a novel membrane distillation process. Journal of Materials Chemistry A, 2018. 6(39): p. 18799-18807.

11. Ye, H., et al., Silver Nanoparticle-Enabled Photothermal Nanofibrous Membrane for Light-Driven Membrane Distillation. Industrial \& Engineering Chemistry Research, 2019. 58(8): p. 3269-3281.

12. Huang, Q., et al., Study on photothermal PVDF/ATO nanofiber membrane and its membrane distillation performance. Journal of Membrane Science, 2019. 582: p. 203-210.

13. Said, I.A., S. Wang, and Q. Li, Field Demonstration of a Nanophotonics-Enabled Solar Membrane Distillation Reactor for Desalination. Industrial \& Engineering Chemistry Research, 2019. 58(40): p. 18829-18835.

14. Wang, P., Emerging investigator series: the rise of nano-enabled photothermal materials for water evaporation and clean water production by sunlight. Environmental Science: Nano, 2018. 5(5): p. 1078-1089.

15. Gong, B., et al., Superstructure-Enabled Anti-Fouling Membrane for Efficient Photothermal Distillation. ACS Sustainable Chemistry \& Engineering, 2019.

16. Alsaati, A. and A.M. Marconnet, Energy efficient membrane distillation through localized heating. Desalination, 2018. 442: p. 99-107.

17. Sui, D., et al., Flexible and transparent electrothermal film heaters based on graphene materials. Small, 2011. 7(22): p. 3186-3192.

18. Jia, S.-L., et al., Carbon nanotube-based flexible electrothermal film heaters with a high heating rate. Royal Society Open Science, 2018. 5(6): p. 172072.

19. Dudchenko, A.V., et al., Frequency-dependent stability of CNT Joule heaters in ionizable media and desalination processes. Nature nanotechnology, 2017. 12(6): p. 557.

20. Li, K., et al., Electrothermal-driven membrane distillation for low energy consumption and wetting mitigation. Environmental Science \& Technology, 2019.

21. Shah, T.K., et al., Carbon nanostructures and methods of making the same. 2014, Google Patents.

22. Susantyoko, R.A., et al., A surface-engineered tape-casting fabrication technique toward the commercialisation of freestanding carbon nanotube sheets. Journal of Materials Chemistry A, 2017. 5(36): p. 19255-19266. 
23. van der Pauw, L.J., A method of measuring the resistivity and Hall coefficient on lamellae of arbitrary shape. Philips technical review, 1958. 20: p. 220-224.

24. Elmarghany, M.R., et al., Thermal analysis evaluation of direct contact membrane distillation system. Case Studies in Thermal Engineering, 2019. 13: p. 100377.

25. Swaminathan, J., et al., Energy efficiency of membrane distillation up to high salinity: Evaluating critical system size and optimal membrane thickness. Applied Energy, 2018. 211: p. 715-734.

26. Soomro, M.I. and W.-S. Kim, Parabolic-trough plant integrated with direct-contact membrane distillation system: Concept, simulation, performance, and economic evaluation. Solar Energy, 2018. 173: p. 348-361.

27. Hashaikeh, R., et al., A novel in situ membrane cleaning method using periodic electrolysis. Journal of Membrane Science, 2014. 471: p. 149-154.

28. Villa, F., M. Marengo, and J. De Coninck, A new model to predict the influence of surface temperature on contact angle. Scientific reports, 2018. 8(1): p. 1-10.

29. Phillips, M.C. and A.C. Riddiford, Temperature Dependence of Contact Angles. Nature, 1965. 205(4975): p. 1005-1006.

30. Gould, R.F., Contact Angle, Wettability, and Adhesion, Copyright, Advances in Chemistry Series, in Contact Angle, Wettability, and Adhesion, F.G. Robert, Editor. 1964, AMERICAN CHEMICAL SOCIETY. p. i-iii.

31. Ebrahimi, F. and M. Gholamian Moghaddam, Temperature-dependence of wetting properties of carbon nanotubes. Physica A: Statistical Mechanics and its Applications, 2016. 453: p. 271-277.

32. Vaitheeswaran, S., J.C. Rasaiah, and G. Hummer, Electric field and temperature effects on water in the narrow nonpolar pores of carbon nanotubes. The Journal of chemical physics, 2004. 121(16): p. 7955-7965.

33. Drioli, E., Membrane Distillation. 2018: MDPI AG.

34. Khayet, M. and T. Matsuura, Chapter 13 - Air Gap Membrane Distillation, in Membrane Distillation, M. Khayet and T. Matsuura, Editors. 2011, Elsevier: Amsterdam. p. 361-398.

35. Khayet, M., Membranes and theoretical modeling of membrane distillation: A review. Advances in Colloid and Interface Science, 2011. 164(1): p. 56-88.

36. Schneider, K., et al., Membranes and modules for transmembrane distillation. Journal of Membrane Science, 1988. 39(1): p. 25-42.

37. Chen, W., et al., High-flux water desalination with interfacial salt sieving effect in nanoporous carbon composite membranes. Nature nanotechnology, 2018. 13(4): p. 345-350.

38. Mustafa, I., et al., Fabrication of Freestanding Sheets of Multiwalled Carbon Nanotubes (Buckypapers) for Vanadium Redox Flow Batteries and Effects of Fabrication Variables on Electrochemical Performance. Electrochimica Acta, 2017. 230: p. 222-235.

39. Shim, W., et al., Optimally conductive networks in randomly dispersed CNT: graphene hybrids. Scientific reports, 2015. 5: p. 16568.

40. Ahmed, F.E., et al., Electrically conducting nanofiltration membranes based on networked cellulose and carbon nanostructures. Desalination, 2017. 406: p. 60-66.

41. Spitalsky, Z., et al., High volume fraction carbon nanotube-epoxy composites. Nanotechnology, 2009. 20(40): p. 405702.

42. de Lannoy, C.F., et al., A highly electrically conductive polymer-multiwalled carbon nanotube nanocomposite membrane. Journal of Membrane Science, 2012. 415-416: p. 718-724.

43. Jakubinek, M., et al. Multifunctional skin materials based on tailorable, carbon-nanotubepolyurethane composite sheets. in 2018 AIAA/ASCE/AHS/ASC Structures, Structural Dynamics, and Materials Conference. 2018.

44. Miao, M., Electrical conductivity of pure carbon nanotube yarns. Carbon, 2011. 49(12): p. 37553761. 
45. Lekawa-Raus, A., et al., Electrical Properties of Carbon Nanotube Based Fibers and Their Future Use in Electrical Wiring. Advanced Functional Materials, 2014. 24(24): p. 3661-3682.

46. Li, C., E.T. Thostenson, and T.-W. Chou, Dominant role of tunneling resistance in the electrical conductivity of carbon nanotube-based composites. Applied Physics Letters, 2007. 91(22): p. 223114.

47. Kulesza, S., et al., High-temperature electrical transport properties of buckypapers composed of doped single-walled carbon nanotubes. Carbon, 2006. 44(11): p. 2178-2183.

48. Sudoh, M., et al., Effects of thermal and concentration boundary layers on vapor permeation in membrane distillation of aqueous lithium bromide solution. Journal of Membrane Science, 1997. 131(1): p. 1-7.

49. Suárez, F. and R. Urtubia, Tackling the water-energy nexus: an assessment of membrane distillation driven by salt-gradient solar ponds. Clean Technologies and Environmental Policy, 2016. 18(6): p. 1697-1712.

50. Khayet, M. and T. Matsuura, Membrane Distillation: Principles and Applications. 2011: Elsevier Science.

51. Alkhudhiri, A., N. Darwish, and N. Hilal, Produced water treatment: Application of Air Gap Membrane Distillation. Desalination, 2013. 309: p. 46-51.

52. Zaragoza, G., J. Andrés-Mañas, and A. Ruiz-Aguirre, Commercial scale membrane distillation for solar desalination. npj Clean Water, 2018. 1(1): p. 1-6.

53. Wang, W., et al., Simultaneous production of fresh water and electricity via multistage solar photovoltaic membrane distillation. Nature communications, 2019. 10(1): p. 1-9.

54. Jansen, A., et al., Development and pilot testing of full-scale membrane distillation modules for deployment of waste heat. Desalination, 2013. 323: p. 55-65.

55. Dow, N., et al., Pilot trial of membrane distillation driven by low grade waste heat: Membrane fouling and energy assessment. Desalination, 2016. 391: p. 30-42. 